\title{
A Technical Note: Inter-Observer and Inter-Modality Variability of Cone-Beam Computed Tomography (CBCT) and Ultrasound (US) in Stereotactic Body Radiotherapy for Kidney Cancer
}

\author{
Ronnie W. K. Leung, Matthew Y. P. Wong, Venus W. Y. Lee, Steven K. T. Cheung, \\ Aray K. L. Wong, Hollis S. L. Luk, Francesca K. L. Ng, Gilbert M. L. Law, Frankle K. H. Lee, \\ W. H. Mui, Dennis Y. K. Ngar \\ Department of Clinical Oncology, Tuen Mun Hospital, Hong Kong, China \\ Email: lwk189@ha.org.hk
}

How to cite this paper: Leung, R.W.K. Wong, M.Y.P., Lee, V.W.Y., Cheung, S.K.T., Wong, A.K.L., Luk, H.S.L., Ng, F.K.L., Law, G.M.L., Lee, F.K.H., Mui, W.H. and Ngar, D.Y.K. (2017) A Technical Note: Inter-Observer and Inter-Modality Variability of Cone-Beam Computed Tomography (CBCT) and Ultrasound (US) in Stereotactic Body Radiotherapy for Kidney Cancer. International Journal of Medical Physics, Clinical Engineering and Radiation Oncology, 6, 392-400.

https://doi.org/10.4236/ijmpcero.2017.64035

Received: September 20, 2017

Accepted: October 31, 2017

Published: November 3, 2017

Copyright $\odot 2017$ by authors and Scientific Research Publishing Inc. This work is licensed under the Creative Commons Attribution International License (CC BY 4.0).

http://creativecommons.org/licenses/by/4.0/

\begin{abstract}
Introduction: To investigate the inter-observer and inter-modality variabilities of two imaging guided equipments-cone-beam computed tomography (CBCT) and ultrasound (US) in kidney stereotactic body radiotherapy. Methods: A renal metastasis case implanted with three gold anchor fiducial markers was firstly scanned by US to acquire a 3-dimension US image and followed by 4 -dimension CBCT in every fraction. Seven observers retrospectively registered the pre-treatment images with the corresponding reference images based on the gold markers. Registration uncertainty of the observers between two imaging modalities was evaluated. Results: The uncertainties over whole treatment course in CBCT were $0.88 \mathrm{~mm}, 1.94 \mathrm{~mm}$ and $0.86 \mathrm{~mm}$ in lateral, longitudinal and vertical directions respectively; while $0.8 \mathrm{~mm}, 0.97 \mathrm{~mm}$ and 1.36 $\mathrm{mm}$ were found in US. Conclusion: The greatest uncertainty was found in longitudinal direction in $\mathrm{CBCT}$ due to the fact that the respiration motion is the most rigorous in cranial-caudal direction. In US, since the probe was hold almost in upright position, the strong echo in vertical direction was attributed to the greatest uncertainty for such direction.
\end{abstract}

Keywords

Inter-Observer, Inter-Modality, Variability, CBCT, US, Kidney SBRT

\section{Introduction}

Stereotactic body radiotherapy (SBRT) has been widely adopted which delivers 
high ablative doses to tumors while sparing as much normal tissue as possible. In the management of renal cell cancer, SBRT can be served as an alternative to surgery and was found to be superior to conventional radiotherapy fractionation regimes (1.8 - 2 Gy) due to its radioresistance in nature [1] [2]. The implementation of image-guidance further facilitates the use of SBRT to provide accurate positioning and targeting of the tumors, which in turn promotes the margin reduction for dose escalation and normal tissue sparing. The advance of image-guided radiotherapy (IGRT) can provide pre-treatment positioning, target monitoring and post-treatment verification using different modalities such as infrared, X-rays, ultrasound and magnetic resonance imaging (MRI) [3]. Ultrasound (US) is a non-ionizing image modality offering better soft tissue contrast than X-ray. Various venders provide different implementation of US into radiotherapy. For example, boost irradiation to a lumpectomy cavity after whole breast radiotherapy was proved to efficiently reduce the risk of local recurrence [4] [5]. Good visualization of the lumpectomy cavity and the tumor bed in US images was reported and potential margin reduction could be achieved by daily US guidance for localization and positioning [6] [7]. In cervical cancer treatment, US can be used for target visualization, catheter placement and even treatment planning in brachytherapy [8] [9]. Although US may not be as helpful as MRI to identify the low-, intermediate- and high-risk clinical target volume (CTV), the real-time feedback and lower amount of resources required are advantages over other modalities. In addition, US has been widely used for prostate cancer treatment [10] [11]. Its accuracy and consistency were compared with other imaging modalities, for instance, cone-beam computed tomography (CBCT) and electromagnetic transponders, where it has proved to be clinically acceptable [12] [13]. Daily variations and real-time tracking of prostates are available and immediate action could be taken if large deviations are detected from the baseline. The implementation of US in other parts of body is under extensive investigation. State-of-the-art robotic arms are being deployed to hold US probes and monitor the targets in SBRT. The Johns Hopkins group has designed a robotic manipulator with a novel cooperative control strategy for soft tissue targeting in upper abdomen [14], reporting less than $2.2 \mathrm{~mm}$ setup error by using US image feedback. Furthermore, robust $4 \mathrm{D}$ robotic US system imaging on the prostate, liver, pancreas and kidneys of healthy volunteers was demonstrated by Stanford group [15]. However, there are very limited studies implementing US for target localization in kidney cancer and also variations and precautions of using US for kidney cancer have not yet been investigated and reported. Therefore, this study aims to investigate the variations of inter-observer and inter-modality of using US and CBCT in kidney SBRT.

\section{Materials and Methods}

A renal cancer patient had three gold anchors implanted under diagnostic US-guidance by an interventional radiologist one week before their planning CT scan. The gold markers were placed at least $1 \mathrm{~cm}$ away from each other at the 
lesion periphery with different angular separations. Automatic breathing control $(\mathrm{ABC})$ was proposed for motion management. Optimal trigger level and breath-hold duration were carefully chosen under fluoroscopic screening for the latter CT simulation stage. Afterwards, the Clarity ultrasound auto-scan probe (Elekta $A B$, Stockholm, Sweden) was held by a physicist at a slightly oblique angle to avoid deformation and displacement of the kidney. Under the same trigger level, a reference 3D-US image was acquired during the breath-hold. Figures 1(a)-(c) shows an US reference image overlaid with the planning $\mathrm{CT}$ in the axial, coronal and sagittal planes. A $5 \mathrm{~mm}$ isotropic planning target volume (PTV) margin was expanded from the CTV to account for potential overshoot issue of the ABC system, day-to-day variations and setup uncertainty. 50 Gy in 5 fractions was prescribed to the PTV and treated with a 6 MV Elekta Agility Linac. However, on the first day of treatment course, the patient's condition deteriorated and found difficulty in tolerating the long treatment time using the $\mathrm{ABC}$ system. Therefore, abdominal compression was adopted with sufficient pressure to reduce the breathing amplitude whilst minimising the deformation of the target and surrounding tissues. A helical four-dimensional computed tomography with $2 \mathrm{~mm}$ slice thickness

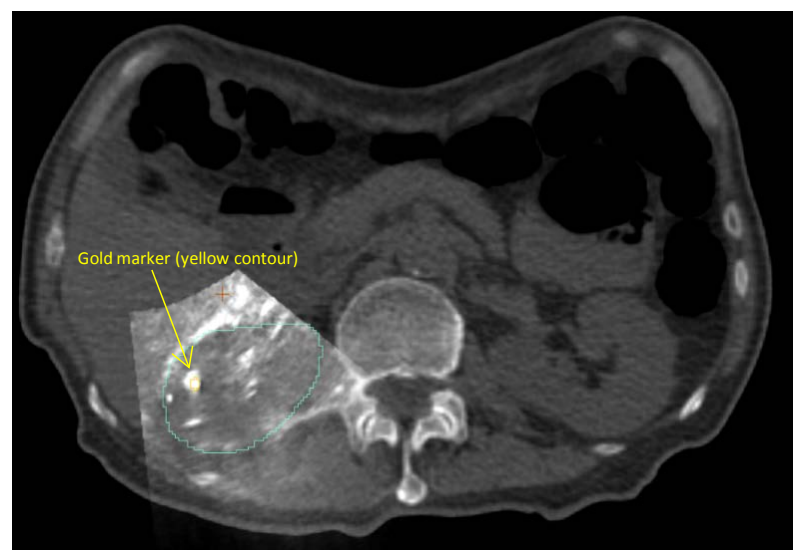

(a)

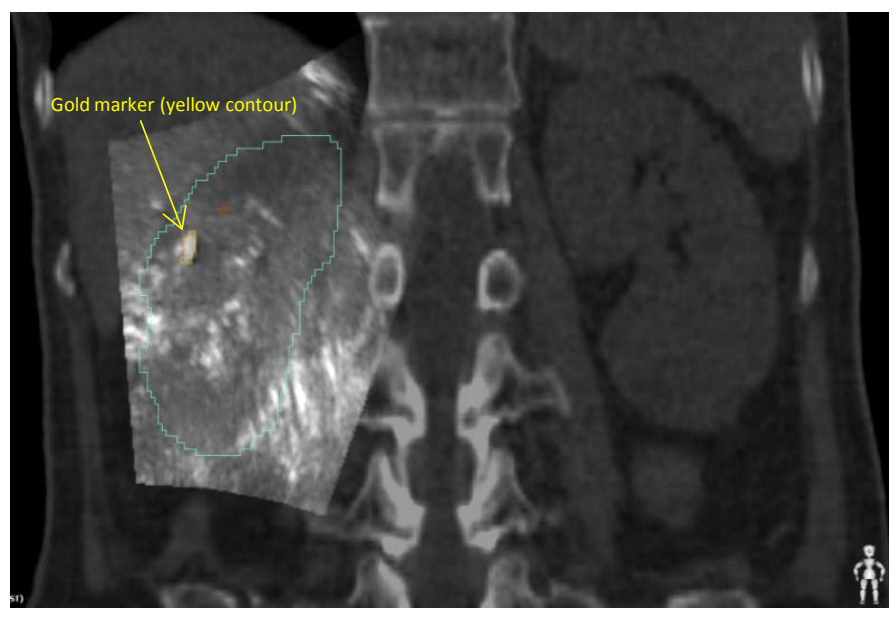

(b)

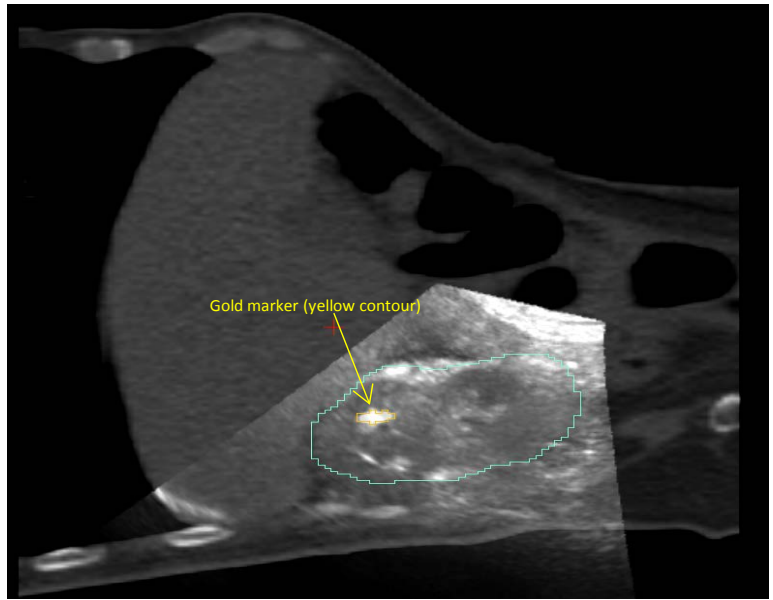

(c)

Figure 1. CT-US overlay in (a) axial; (b) coronal; and (c) sagittal planes. Yellow structure represents one of the markers and blue one is the right kidney contoured based on planning CT. 
was acquired. Mid-ventilation (MidV) phase was determined from the ten time-binned CT images and the Van Herk margin formula was used to generate the PTV. Since the US reference image set was obtained over one week prior to the CT scan, in order to avoid any discrepancy of the target due to rapid deteriorated health condition, a new reference US acquisition was conducted on the first treatment day in the treatment room. In the remaining fractions, daily 3D-US was acquired for retrospective analysis. Translational couch correction was applied according to the average phase from the 4D-CBCT registered with MidV planning CT in the X-ray Volumetric Imager (XVI) system. Seven physicists and therapists retrospectively registered the US and CBCT images with the corresponding reference images. In the US registration case, the sound wave was reflected at the interface between the gold marker and soft tissue due to the difference in acoustic impedance. The signals enhanced at this region became an excellent surrogate and was assigned as a planning reference volume (PRV) to allow the observers to perform the registration (Figure 2). In the X-ray modality, automatic image registration based on seeds (gold markers) was performed and followed by manual fine adjustment (Figure 3). Inter-observer and inter-modality variations were then compared.

\section{Results}

After manual fine adjustments following automatic 4D image registration in XVI, the averaged seven observers' variations over the whole treatment course were $0.88 \mathrm{~mm}, 1.94 \mathrm{~mm}$ and $0.86 \mathrm{~mm}$ in lateral, longitudinal and vertical directions respectively (Table 1 ). While from the retrospective registration using US images, the variations were $0.8 \mathrm{~mm}, 0.97 \mathrm{~mm}$ and $1.36 \mathrm{~mm}$ in lateral, longitudinal and vertical directions respectively (Table 1). Additional information of daily displacement from the baselines of two different imaging systems was also analysed, although only one patient was involved in this study. In each imaging system, the positional errors of seven observers for each fraction were averaged as a more representable value $(\mathrm{M})$ for that particular fraction. The fractional values $M_{C B C T}$ (averaged values for CBCT) and $M_{U S}$ (averaged values for Clarity US) were shown in Table 2 and Table 3.

\section{Discussion}

Non-ionizing US-guidance is commonly used in prostate cancer treatment to provide comparable accuracy as CBCT. The difference of setup errors between US and CBCT was extensively investigated and was found to agree within $3 \mathrm{~mm}$. Fargier et al. compared the inter-observer variations of trans-abdominal US against CBCT for prostate and found these to be 2.2 and $1.1 \mathrm{~mm}$ laterally, 2.6 and $1.8 \mathrm{~mm}$ longitudinally and 2.1 and $2.0 \mathrm{~mm}$ vertically for US and CBCT respectively [16]. Lediju et al. extended the use of a robotic US probe for lower abdomen in an animal study and reported that the average $3 \mathrm{D}$ reproducibility of their robotic US probe was less than $2 \mathrm{~mm}$ [17]. Moreover, the feasibility of implementing US imaging into liver SBRT for eleven patients was shown by Gurp 


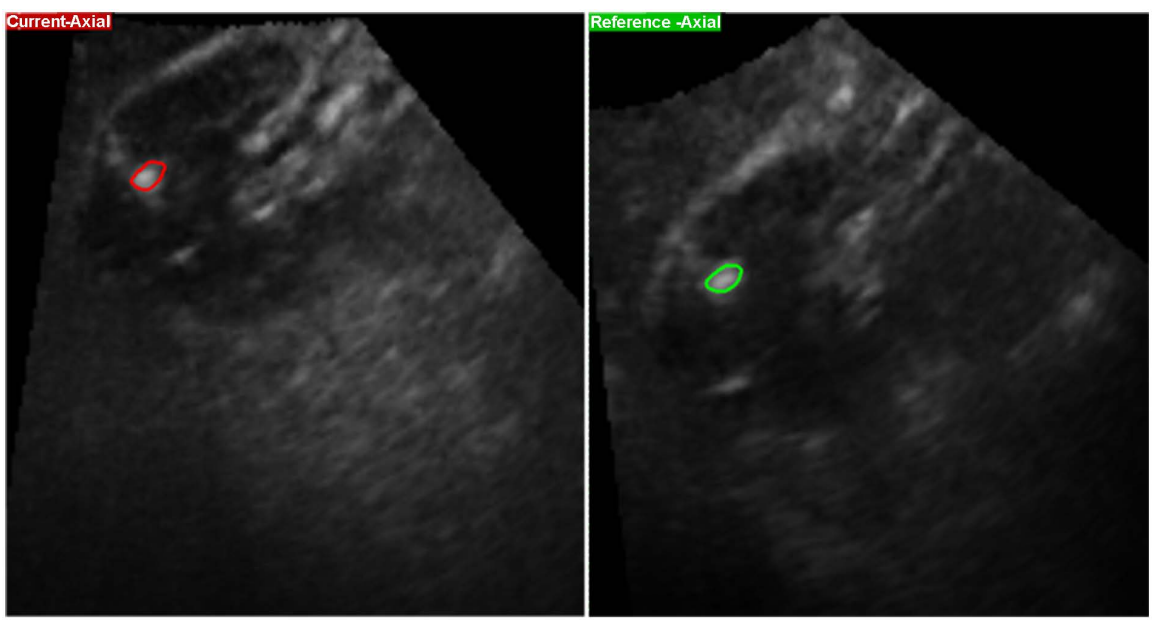

(a)
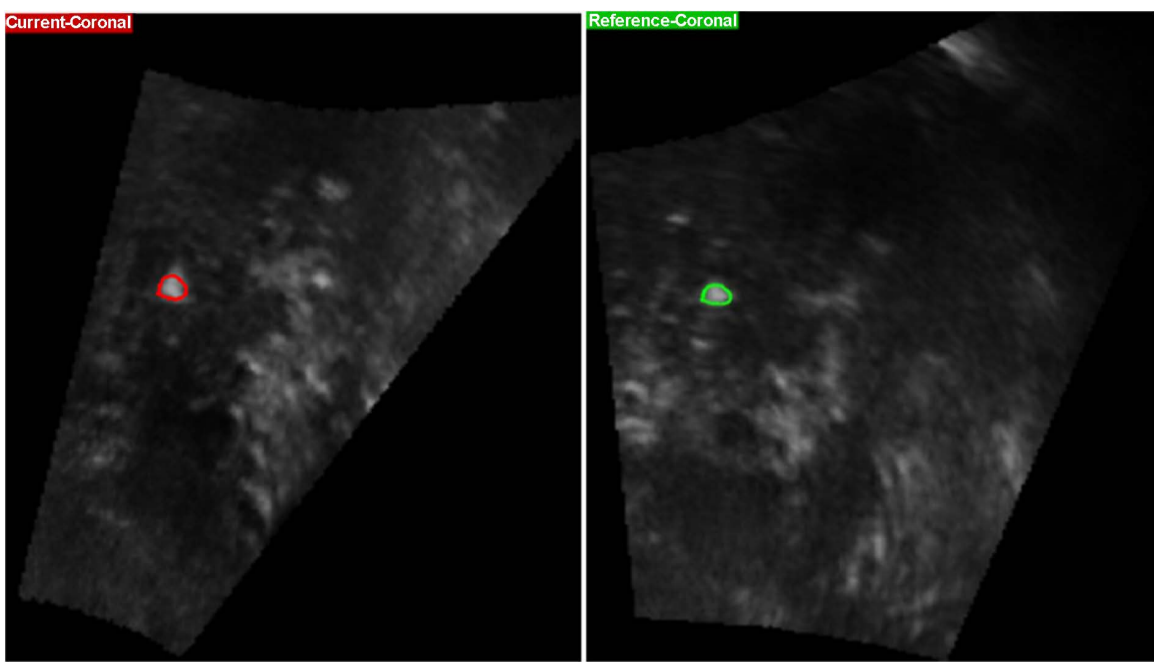

(b)
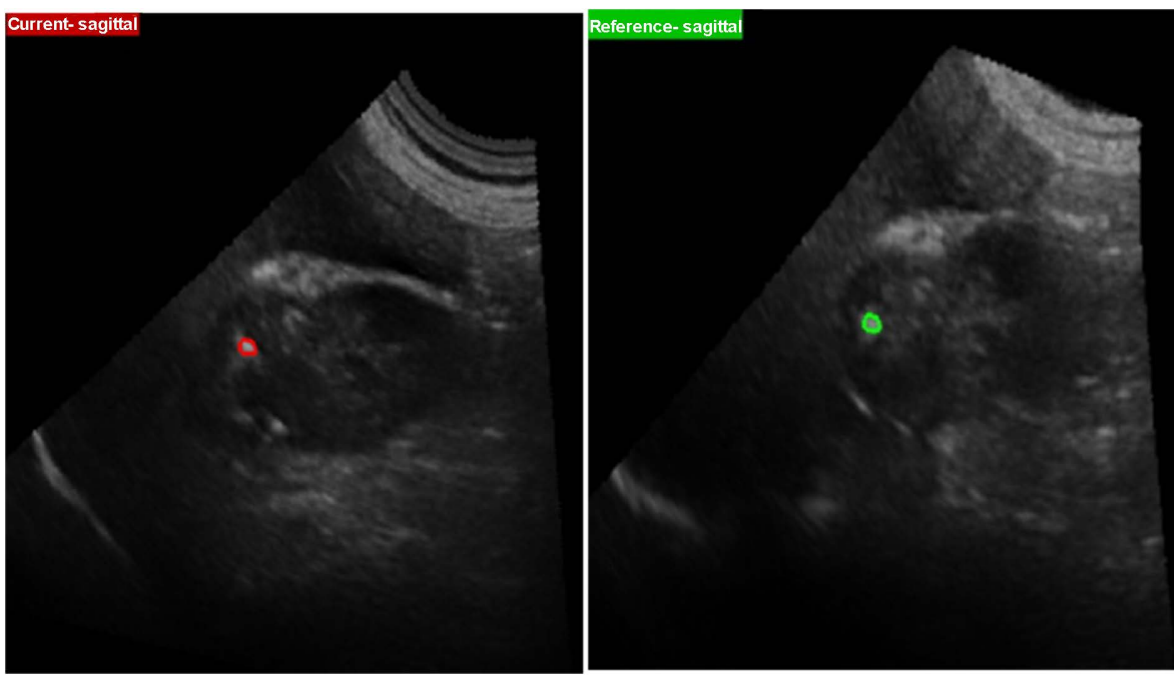

(c)

Figure 2. Daily (left) and reference (right) US images in (a) axial; (b) coronal; (c) sagittal; red and green PRVs are the surrogates for target localization (echoes from the interface between markers and soft tissues). 


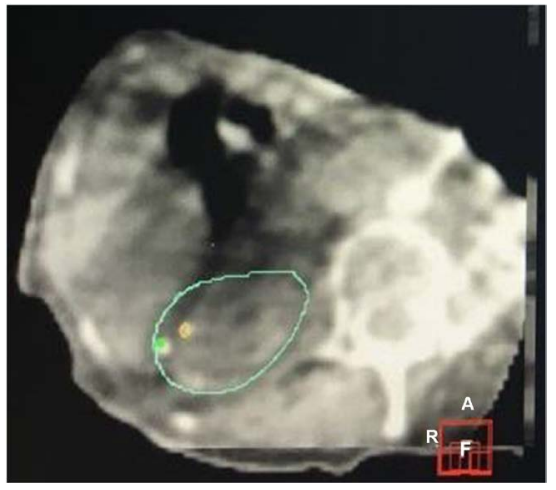

(a)

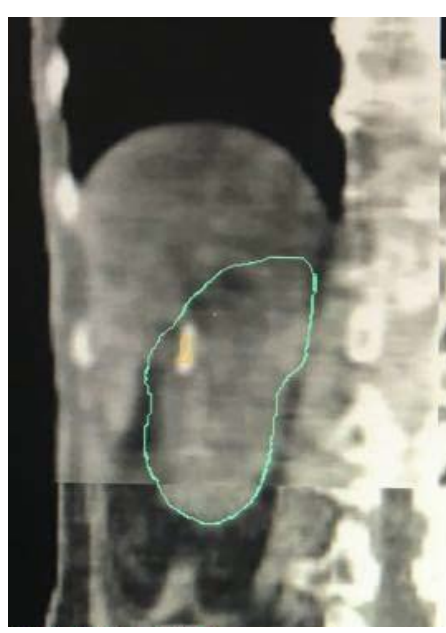

(b)
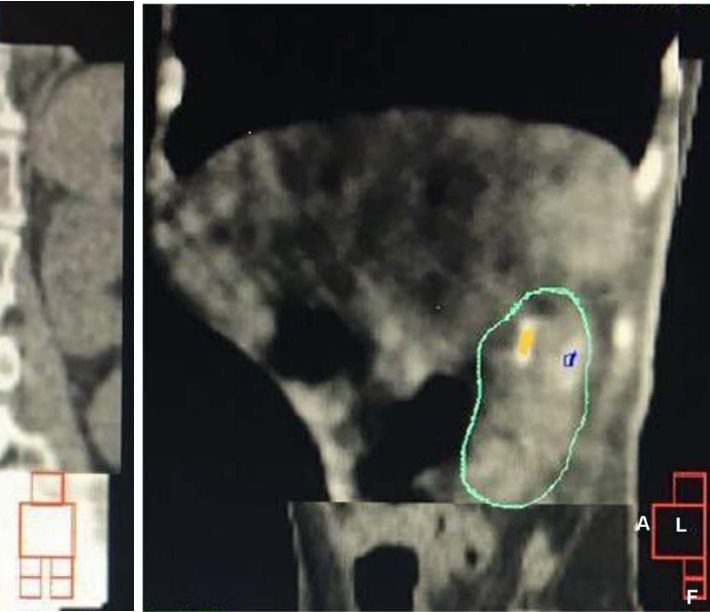

(c)

Figure 3. Mid-ventilation CBCT image registration in (a) axial; (b) coronal and (c) sagittal plan. The yellow, green and blue structures are contoured in planning CT presenting the gold markers.

Table 1. Average of seven observers' variabilities of two different imaging modalities.

\begin{tabular}{cccc}
\hline & Lateral $(\mathrm{mm})$ & Longitudinal $(\mathrm{mm})$ & Vertical $(\mathrm{mm})$ \\
\hline CBCT & 0.88 & 1.94 & 0.86 \\
Clarity US & 0.80 & 0.97 & 1.36 \\
\hline
\end{tabular}

Table 2. Daily displacement from the baseline in CBCT $M_{C B T B}$.

\begin{tabular}{lccc}
\hline & Lateral $(\mathrm{mm})$ & Longitudinal $(\mathrm{mm})$ & Vertical $(\mathrm{mm})$ \\
\hline Fraction 2 & 3.8 & 2.4 & -0.5 \\
Fraction3 & 4.3 & 7.3 & -1.0 \\
Fraction4 & 1.5 & 3.1 & 0.8 \\
Fraction5 & 4.8 & -2.7 & -1.5 \\
\hline
\end{tabular}

et al. and reported the inter-observer variations were $1.6 \mathrm{~mm}, 2.8 \mathrm{~mm}$ and $1.2 \mathrm{~mm}$ in lateral, longitudinal and vertical directions respectively [18]. To our knowledge, 
Table 3. Daily displacement from the baseline in US $M_{U S}$

\begin{tabular}{cccc}
\hline & Lateral $(\mathrm{mm})$ & Longitudinal $(\mathrm{mm})$ & Vertical $(\mathrm{mm})$ \\
\hline Fraction 2 & 5.3 & 3.6 & -1.1 \\
Fraction3 & 4.7 & 3.1 & 0.9 \\
Fraction4 & 1.6 & -2.8 & -0.5 \\
Fraction5 & 1.2 & -4.0 & -0.2 \\
\hline
\end{tabular}

this study is the first to investigate and compare the intra- and inter-observer variations between US and CBCT system in kidney cancer. Our results demonstrated that the largest variation is in the vertical direction $(1.36 \mathrm{~mm})$ and less than $1 \mathrm{~mm}$ for the other two directions, which was expected to be smaller than the findings from Gurp group in which breathing motion was managed with free-breathing and active breathing control. Another interesting result from $\mathrm{Ta}$ ble 1 was the different directions for the largest variation in two imaging modalities (1.94 $\mathrm{mm}$ in longitudinal direction in CBCT and $1.36 \mathrm{~mm}$ in vertical direction in US). This is because $4 \mathrm{D}$ CBCT was utilized which introduced the largest uncertainty in the longitudinal direction due to respiratory motion during reconstruction. Elongated gold seeds could be observed in Figure 3 when comparing those in planning CT. In the case of US, as the acoustic beam was almost in an upright direction, the strong echo (surrogate) due to the different acoustic properties between gold seed and soft tissue in this direction (a long "tail" just below the surrogate as shown in Figure 2(a) and Figure 2(c)) was the main reason of being the largest uncertainty in vertical direction. Nonetheless, both imaging systems showed acceptable variations in all directions. Another finding from Table 2 and Table 3 showed that similar displacement from the baselines using the two imaging modalities was obtained except for the longitudinal direction. This could be explained by the fact that respiratory motion was involved during CBCT while the breath-hold technique was used in US.

From our results, different sources of error exist in both imaging system. Therefore, hybrid image-guided system was recommended to provide more information and accurate positioning. Similar recommendation was made by Kroll's study from which better visualization of salivary gland could be achieved by using CBCT as an adjunct to US [19]. One of the feasible workflow would be using US for initial setup to reduce the positioning time and verified by CBCT or other X-ray system [20]. One limitation of this study was the patient size which served as the further goal to increase the sample size from which a population-based margin for kidney diseases could be established.

\section{Conclusion}

This study showed that the Clarity US system is able to provide volumetric localization in kidney SBRT. Moreover, our results illustrated that the inter-observer variations in two different imaging modalities were comparable and acceptable. 
Further study of US image-guidance in kidney SBRT in a large patient cohort is warranted.

\section{References}

[1] Blanco, A.I., Teh, B.S. and Amato, R.J. (2011) Role of Radiation Therapy in the Management of Renal Cell Cancer. Cancers, 3, 4010-4023.

https://doi.org/10.3390/cancers3044010

[2] Rule, W.G., Stanic, S., Boike, T.P. and Timmerman, R.D. (2011) Is Renal Cell Carcinoma Really Radioresistant? Experience with Stereotactic Body Radiotherapy in Patients for Primary and Metastatic Renal Cell Carcinoma. Kidney Cancer Journal, 9, 35-40.

[3] Goyal, S. and Kataria, T. (2014) Image Guidance in Radiation Therapy: Techniques and Application. Radiology Research and Practice, 2014, Article ID: 705604. https://doi.org/10.1155/2014/705604

[4] Bartelink, H., Horiot, J.C. and Poortmans, P. (2001) Recurrence Rates after Treatment of Breast Cancer with Standard Radiotherapy with or without Additional Radiation. New England Journal of Medicine, 345, 1378-1387. https://doi.org/10.1056/NEJMoa010874

[5] Bartelink, H., Horiot, J.C. and Poortmans, P. (2007) Impact of a Higher Radiation Dose on Local Control and Survival in Breast-Conserving Therapy of Early Breast Cancer: 10-Year Results of the Randomized Boost versus on Boost EORTC 22881-10882 Trial. Journal of Clinical Oncology, 25, 3259-3265. https://doi.org/10.1200/JCO.2007.11.4991

[6] Wong, D., Muanza, T., Reynard, E., Barker, J., Robert, K. and Sultanem, K. (2009) Use of 3D-Ultrasound in the Detection of Breast Tumor Bed Displacement during Radiotherapy. International Journal of Radiation Oncology Biology Physics, 75, S196-S197.

[7] Heimann, R., Hard, D. and Archambault, J. (2009) Three Dimensional Ultrasound (3DUS) Image Guidance, Clips or CT: How to Optimally Localize the Beast Lumpectomy Cavity Volume? International Journal of Radiation Oncology Biology Physics, 75, S196.

[8] Kamrava, M. (2014) Potential Role of Ultrasound Imaging in Interstitial Image Based Cervical Cancer Brachytherapy. Journal of Contemporary Brachytherapy, 6, 223-230. https://doi.org/10.5114/jcb.2014.43778

[9] Narayan, K., van Dyk, S., Bernshaw, D., Khaw, P., Mileshkin, L. and Kondalsamy-Chennakesavan, S. (2014) Ultrasound Guided Conformal Brachytherapy of Cervix Cancer: Survival, Patterns of Failure, and Late Complications. Journal of $\mathrm{Gy}^{-}$ necologic Oncology, 25, 206-213. https://doi.org/10.3802/jgo.2014.25.3.206

[10] Bake, M. and Behrens, C.F. (2016) Determining Intrafractional Prostate Motion using Four Dimensional Ultrasound System. BMC Cancer, 16, 484. https://doi.org/10.1186/s12885-016-2533-5

[11] Robinson, D., Liu, D., Steciw, S., Field, C., Daly, H., Saibishkumar, E.P., Fallone, G., Parliament, M. and Amanie, J. (2012) An Evaluation of the Clarity 3D Ultrasound System for Prostate Localization. Journal of Applied Clinical Medical Physics, 13, 100-112. https://doi.org/10.1120/jacmp.v13i4.3753

[12] Li, M., Ballhausen, H., Hegemann, N.S., et al. (2015) A Comparative Assessment of Prostate Positioning Guided by Three-Dimensional Ultrasound and Cone Beam CT. Radiation Oncology (London, England), 10, 82.

https://doi.org/10.1186/s13014-015-0380-1 
[13] Abramowitz, M.C., Bossart, E., Flook, R., Wu, X., Brooks, R., Lachaine, M., et al. (2012) Noninvasive Real-Time Prostate Tracking using a Transperineal Ultrasound Approach. International Journal of Radiation Oncology Biology Physics, 84, S133.

[14] TutkunŞen, H., Lediju Bell, M.A., Iordachita, I., Wong, J. and Kazanzides, P. (2013) A Cooperatively Controlled Robot for Ultrasound Monitoring of Radiation Therapy. Proceedings of the IEEE/RSJ International Conference on Intelligent Robots and Systems, 3071-3076.

[15] Western, C., Hristov, D. and Schlosser, J. (2015) Ultrasound Imaging in Radiation Therapy: From Interfractional to Intrafractional Guidance. Cureus, 7, e280. https://doi.org/10.7759/cureus.280

[16] Marie, F.-V., et al. (2015) Ultrasound versus Cone-Beam CT Image-Guided Radiotherapy for Prostate and Post-Prostatectomy Pretreatment Localization, Physica Medica.

[17] Lediju Bell, M.A., Sen, H.T., Iordachita, I., Kazanzides, P. and Wong, J. (2014) In Vivo Reproducibility of Robotic Probe Placement for a Novel Ultrasound-Guided Radiation Therapy System. Journal of Medical Imaging, 1, Article ID: 025001.

[18] Bloemen-van Gurp, E., Van Der Meer, S., Hendry, J., et al. (2013) Active Breathing Control in Combination with Ultrasound Imaging: A Feasibility Study of Image Guidance in Stereotactic Body Radiation Therapy of Liver Lesions. International Journal of Radiation Oncology, Biology, Physics, 85, 1096-1102.

[19] Kroll, T., May, A., Wittekindt, C., et al. (2015) Cone Beam Computed Tomography (CBCT) Sialography-An Adjunct to Salivary Gland Ultrasonography in the Evaluation of Recurrent Salivary Gland Swelling. Oral Surgery, Oral Medicine, Oral Pathology, Oral Radiology, 120, 771-775.

[20] Scarbrough, T.J., Golden, N.M., Ting, J.Y., et al. (2006) Comparison of Ultrasound and Implanted Seed Marker Prostate Localization Methods: Implication for Image-Guided Radiotherapy. International Journal of Radiation Oncology, Biology, Physics, 65, 378-387. 